\title{
ON THE OPTIMAL DESIGN OF A VIBRATING BEAM*
}

\author{
BY \\ FRITHIOF I. NIORDSON \\ The Royal Technical University of Denmark, Copenhagen
}

\begin{abstract}
This paper deals with the problem of finding the best possible tapering of a simply supported beam, which for a given volume would have the highest possible value of the natural frequency for the lowest mode of lateral vibrations. The differential equation governing the deflection is given and a procedure to solve this non-linear
\end{abstract} equation by successive iterations is presented.

1. Introduction. The interest in optimal design of columns and beams has existed for many years. A special group of optimal design problems are connected with eigenvalue problems, namely those in which stability or vibrations have to be considered. The classical problem of finding the optimal shape of an Euler-Bernoulli column, subjected to a compressive load was treated by Lagrange [1] in 1770, whose treatment of this subject, however, is faulty. The correct solution was first obtained by Clausen [2] in 1851 and then, independently by Keller [3] in 1960. It was found that by tapering the column, the critical load could be increased at most by $\frac{1}{3}$ in comparison with a cylindrical column of the same material, volume, length and cross-sectional form. This result was later on extended by Tadjbakhsh and Keller [4] to include other boundary conditions and some additional results. Beesack [5] and Schwarz [6], [7], [8] have studied the extreme values of the natural frequencies of strings, beams, and plates in which cases the mass distribution was varied while everything else was held constant.

In this paper we shall deal with the optimal design of a vibrating beam, assuming a certain relation between the mass per unit length and the bending rigidity.

A simply supported beam, performing small harmonic vibrations in a plane, will have an infinite number of natural frequencies. We shall restrict our attention to the lowest frequency $\omega_{0}$. Let us assume that all cross sections are similar and of a given form, and that, furthermore, the material, length, and volume of the beam are given. What tapering will then give the beam the highest possible value of $\omega_{0}$ ?

It will be shown that the lowest natural frequency $\omega_{0}$ for the most appropriately tapered beam will exceed that of a cylindrical beam of the same material, length, volume, and cross-sectional form only by approximately 6.6 per cent.

The result can also be stated as an isoperimetric inequality, stating that the lowest natural frequency of a beam of a given material and cross-sectional form is always less or equal to a certain function of its length and volume.

Apart from its theoretical interest, this result may have some importance in the design of shafts, where it shows how much, or rather, how little can be won in critical speed by tapering the shaft.

2. Bending vibrations of a tapered beam. Let us consider small plane vibrations of a straight, simply supported beam. Neglecting the influence of shear stresses and

${ }^{*}$ Received June 26, 1964. 
of rotational inertia, the equation of motion is given by

$$
\frac{d^{2}}{d \xi^{2}}\left(E I \frac{d^{2} y}{d \xi^{2}}\right)-\omega^{2} \gamma A y=0,
$$

where $y$ is the lateral deflection in the plane of bending, $\xi$ the coordinate along the axis of the beam, $E$ Young's modulus, $I$ the principal moment of inertia of the cross section, perpendicular to the plane of bending, $\omega$ the natural angular frequency, $\gamma$ the mass per unit volume, and $A$ the area of the cross section.

In our case, we shall assume that $I$ and $A$ are functions of the coordinate $\xi$. For a simply supported beam, we have the following boundary conditions,

$$
y(0)=0, \quad y(l)=0, \quad E I \frac{d^{2} y}{d \xi^{2}}(0)=0, \quad E I \frac{d^{2} y}{d \xi^{2}}(l)=0,
$$

where $l$ denotes the length of the beam.

Introducing dimensionless quantities, we may write the differential equation and the boundary conditions as follows:

$$
\begin{gathered}
\left(\alpha^{2} y^{\prime \prime}\right)^{\prime \prime}-\lambda \alpha y=0, \\
y(0)=y(1)=\alpha^{2} y^{\prime \prime}(0)=\alpha^{2} y^{\prime \prime}(1)=0 .
\end{gathered}
$$

Here, the dash indicates differentiation with respect to the dimensionless coordinate $x=\xi / l$ while $\alpha(x)$ is the dimensionless area function $\alpha=A l / V$, the total volume of the beam being $V$, and

$$
\lambda=\omega^{2} \frac{\gamma l^{5}}{E c V},
$$

where $c=I / A^{2}$ is a constant, characteristic of the cross sectional form.

From the definition of the area function $\alpha$, it follows that

$$
\int_{0}^{1} \alpha d x=1
$$

The eigenvalues of the problem (2.1), (2.2) are all dependent on and uniquely determined by the function $\alpha(x)$. Let from now on $\lambda$ correspond to the lowest eigenvalue and $y$ to the associated eigenfunction. We now raise the following question. Among all non-negative functions $\alpha$, satisfying the condition (2.4), is there one which renders $\lambda$ a maximum? If so, our problem is to determine this function and to find corresponding eigenvalue $\lambda$.

3. The variational problem. Let us multiply both sides of equation (2.1) by $y$ and integrate between the limits 0 and 1 . If the first term is integrated by parts and the boundary conditions considered, we obtain the well known formula,

$$
\lambda=\frac{\int_{0}^{1} \alpha^{2}\left(y^{\prime \prime}\right)^{2} d x}{\int_{0}^{1} \alpha y^{2} d x}
$$

We assume that our problem has a solution $\alpha(x)$ and that the corresponding first eigenfunction is $y(x)$. Let $\alpha(x, \epsilon)$ be a family of functions depending differentiably on 
the parameter $\epsilon$, all satisfying the condition (2.4) and containing among them the solution $\alpha(x)=\alpha(x, 0)$.

To each function $\alpha(x, \epsilon)$ of this family there corresponds a first eigenfunction $y(x, \epsilon)$, being the solution of the eigenvalue problem. We note that also $y(x, \epsilon)$ will depend differentiably on $\epsilon$ and that furthermore each function $y(x, \epsilon)$ is an "admissible function" to the eigenvalue problem with $\alpha=\alpha(x, 0)$. This follows from the fact that each function $y(x, \epsilon)$ satisfies the essential boundary conditions, i.e. $y(0, \epsilon)=y(1, \boldsymbol{\epsilon})=0$ for all $\epsilon$ and that $y$ is sufficiently differentiable with respect to $x$.

Let us consider the function $R(\epsilon, \delta)$, defined by the quotient

$$
R(\epsilon, \delta)=\frac{\int_{0}^{1}[\alpha(x, \epsilon)]^{2}\left[y^{\prime \prime}(x, \delta)\right]^{2} d x}{\int_{0}^{1} \alpha(x, \epsilon)[y(x, \delta)]^{2} d x} .
$$

Clearly $R(\epsilon, \epsilon)=\lambda(\epsilon)$ is the lowest eigenvalue for any area function of the selected family and $R(0,0)=\lambda(0)$ is the extreme value looked for. Hence,

$$
\left(\frac{d \lambda}{d \epsilon}\right)_{0}=0
$$

or

$$
\left(\frac{d R}{d \epsilon}\right)_{0}=\left(\frac{\partial R}{\partial \epsilon}\right)_{0}+\left(\frac{\partial R}{\partial \delta}\right)_{0}\left(\frac{d \delta}{d \epsilon}\right)_{0}=0,
$$

where index zero denotes the case $\epsilon=\delta=0$.

For $\epsilon=0$ expression (3.2) represents the Rayleigh quotient for the eigenvalue problem (2.1), (2.2). In the domain of all admissible functions, this quotient is minimized by the eigenfunction $y(x)$. Since the functions $y(x, \delta)$ are admissible and contain the solution $y(x, 0)$ among them, it follows that $(\partial R / \partial \delta)_{0}=0$. Thus, condition (3.3) can be replaced by a simpler one,

$$
\left(\frac{\partial R}{\partial \epsilon}\right)_{0}=0
$$

Applying this condition to (3.2), we obtain

$$
\int_{0}^{1} \alpha y^{2} d x \int_{0}^{1} 2 \alpha \alpha_{\epsilon}\left(y^{\prime \prime}\right)^{2} d x-\int_{0}^{1} \alpha^{2}\left(y^{\prime \prime}\right)^{2} d x \int_{0}^{1} \alpha_{\epsilon} y^{2} d x=0,
$$

where $\alpha_{\epsilon}$ denotes the partial derivative of $\alpha$ with respect to $\epsilon$ at $\epsilon=0$.

Dividing through by $\int_{0}^{1} \alpha y^{2} d x$ and making use of (3.1), we get

$$
\int_{0}^{1}\left[2 \alpha\left(y^{\prime \prime}\right)^{2}-\lambda y^{2}\right] \alpha_{\epsilon} d x=0 .
$$

This relation must hold for any particular family of functions $\alpha(x, \epsilon)$ that satisfies condition (2.4), i.e. for which

$$
\int_{0}^{1} \alpha_{\epsilon} d x=0
$$


but this can only be true if the factor $2 \alpha\left(y^{\prime \prime}\right)^{2}-\lambda y^{2}$ is independent of $x$. We may therefore write

$$
2 \alpha\left(y^{\prime \prime}\right)^{2}-\lambda y^{2}=\lambda a^{2}
$$

where $a^{2}$ is a number, independent of $x$. Solving for $\alpha$ we get

$$
\alpha=\frac{\lambda}{2} \frac{a^{2}+y^{2}}{\left(y^{\prime \prime}\right)^{2}}
$$

Substitution into (2.1) yields the following differential equation for $y$ :

$$
\left[\frac{\left(a^{2}+y^{2}\right)^{2}}{\left(y^{\prime \prime}\right)^{3}}\right]^{\prime \prime}=2 \frac{a^{2}+y^{2}}{\left(y^{\prime \prime}\right)^{2}} y \text {. }
$$

To determine $a^{2}$, we multiply Eq. (3.6) by $\alpha$ and integrate between the limits 0 and 1, obtaining

$$
\int_{0}^{1} 2 \alpha^{2}\left(y^{\prime \prime}\right)^{2} d x-\lambda \int_{0}^{1} \alpha y^{2} d x=\lambda a^{2} \int_{0}^{1} \alpha d x .
$$

Substituting from (2.4) and (3.1), we find

$$
a^{2}=\int_{0}^{1} \alpha y^{2} d x
$$

this relation shows that $a^{2}$ is a positive number. Let us now substitute $\alpha$ from (3.7) into (2.4) and (3.9). There result the equations:

$$
\begin{gathered}
a^{2}=a^{2} \frac{\lambda}{2} \int_{0}^{1} \frac{y^{2}}{\left(y^{\prime \prime}\right)^{2}} d x+\frac{\lambda}{2} \int_{0}^{1} \frac{y^{4}}{\left(y^{\prime \prime}\right)^{2}} d x, \\
1=a^{2} \frac{\lambda}{2} \int_{0}^{1} \frac{d x}{\left(y^{\prime \prime}\right)^{2}}+\frac{\lambda}{2} \int_{0}^{1} \frac{y^{2}}{\left(y^{\prime \prime}\right)^{2}} d x,
\end{gathered}
$$

from which $a^{2}$ and $\lambda$ may be obtained as follows:

$$
\begin{gathered}
a^{4}=\frac{\int_{0}^{1}\left(y^{2} / y^{\prime \prime}\right)^{2} d x}{\int_{0}^{1}\left(1 / y^{\prime \prime 2}\right) d x}, \\
\lambda=\frac{2}{\int_{0}^{1}\left(y / y^{\prime \prime}\right)^{2} d x+\left[\int_{0}^{1}\left(y^{2} / y^{\prime \prime}\right)^{2} d x \int_{0}^{1}\left(1 / y^{\prime \prime 2}\right) d x\right]^{1 / 2}} .
\end{gathered}
$$

Since $a^{2}$ is a positive number, the boundary conditions can be written in the form

$$
y(0)=y(1)=\frac{1}{y^{\prime \prime}(0)}=\frac{1}{y^{\prime \prime}(1)}=0 .
$$

The problem to be solved is a non-linear eigenvalue problem consisting of the homogeneous fourth order non-linear differential equation (3.8) and the boundary conditions (3.12).

4. Behaviour of the solution near the end points. The boundary conditions (3.12) 
indicate that the solution is singular at the end points. In order to investigate this singular behavior of the solution, the differential equation (3.8) is written in the form,

$$
y^{\prime \prime \prime \prime}-4 \frac{\left(y^{\prime \prime \prime}\right)^{2}}{y^{\prime \prime}}-\frac{2 y\left(y^{\prime \prime}\right)^{2}+4 y^{\prime \prime}\left(y^{\prime}\right)^{2}-24 y y^{\prime} y^{\prime \prime \prime}}{3\left(a^{2}+y^{2}\right)}-\frac{8 y^{\prime \prime}\left(y y^{\prime}\right)^{2}}{3\left(a^{2}+y^{2}\right)^{2}}=0 .
$$

Let us assume that the solution $y(x)$ can be expanded at $x=0$ in a power series of $x$, such that the lowest non-integer power is $p$. When we substitute $y=b x^{p}+\cdots$ into equation (4.1) and equate the coefficients of the leading terms to zero, we obtain $p=5 / 3$.

The solution thus takes the following form,

$$
y(x)=a_{1} x+a_{2} x^{2}+\cdots+b x^{5 / 3}+\cdots .
$$

which satisfies the boundary conditions at $x=0$.

Substitution into (3.7) shows that the area function $\alpha$ is proportional to $x^{2 / 3}$ for small values of $x$, and thus the linear dimensions-or diameter-of the cross section is proportional to $x^{1 / 3}$ near the endpoint. Clearly, corresponding relations hold at the other end $x=1$.

5. Solution by successive iterations. Since a solution of the differential equation (3.8) cannot be obtained in closed form, we proceed to solve it by successive iterations.

Let us assume that the solution $y(x)$ is symmetric with respect to $x=\frac{1}{2}$. Instead of the boundary conditions at $x=1$, we then have the following at the mid point

$$
y^{\prime}(1 / 2)=\left[\frac{\left(a^{2}+y^{2}\right)^{2}}{\left(y^{\prime \prime}\right)^{3}}\right]_{x=1 / 2}^{\prime}=0 .
$$

By formal integration of (3.8) we find

$$
y(x)=\int_{0}^{x} \int_{x}^{1 / 2}\left[\frac{\left(a^{2}+y^{2}\right)^{2}}{2 \int_{0}^{x} \int_{x}^{1 / 2}\left[\left(a^{2}+y^{2}\right) y / y^{\prime \prime 2}\right] d x^{2}}\right]^{1 / 3} d x^{2},
$$

from which expression we construct a procedure for successive iterations as follows:

$$
y_{n+1}(x)=\int_{0}^{x} \int_{x}^{1 / 2}\left[\frac{\left(a_{n}^{2}+y_{n}^{2}\right)^{2}}{2 \int_{0}^{x} \int_{x}^{1 / 2}\left[\left(a_{n}^{2}+y_{n}^{2}\right) y_{n} / y_{n}^{\prime \prime 2}\right] d x^{2}}\right]^{1 / 3} d x^{2}
$$

where

$$
a_{n}^{4}=\frac{\int_{0}^{1}\left(y_{n}^{2} / y_{n}^{\prime \prime}\right)^{2} d x}{\int_{0}^{1}\left(1 / y_{n}^{\prime \prime 2}\right) d x} .
$$

Starting out with an arbitrary function $y_{0}$ and substituting it into the right hand side of equations (5.3) and (5.2), we obtain a new function $y_{1}$ which satisfies all boundary conditions. In this manner we obtain a sequence of functions, $y_{0}, y_{1}, y_{2}, \cdots$ of which all-except perhaps $y_{0}$-satisfy the boundary conditions. If this sequence converges to a function, that function will satisfy (5.1) and is thus the solution $y(x)$.

Numerical computations indicate that the convergence is very strong. Thus, starting 
out with $y_{0}^{\prime \prime} \equiv 1$ we obtain after only a few iterations an accurate estimate of the solution $y(x)$. The result, the function $y$ and its two first derivatives are shown in Fig. 1. Corresponding value of $\lambda$ is $\lambda=110.6580$. In Fig. 2 the diameter, i.e. $\alpha^{1 / 2}$ is shown as a function of $x$. The dotted line indicates the dimensions of a cylindrical beam of the same volume.

The result can be stated in the following isoperimetric inequality: The lowest natural frequency $\omega_{0}$ of any simply supported beam having similar cross sections satisfies the following inequality,

$$
\omega_{0}^{2} \leq 110.6580 E_{c} V / \gamma l^{5}
$$

Since for a cylindrical beam we have $\lambda_{c}=\pi^{4}$, we conclude that the most appropriate tapering-keeping the total volume unchanged-will increase the lowest natural frequency with a fraction

$$
(110.6580)^{1 / 2} / \pi^{2}=1.066,
$$

i.e. with about 6.6 per cent.

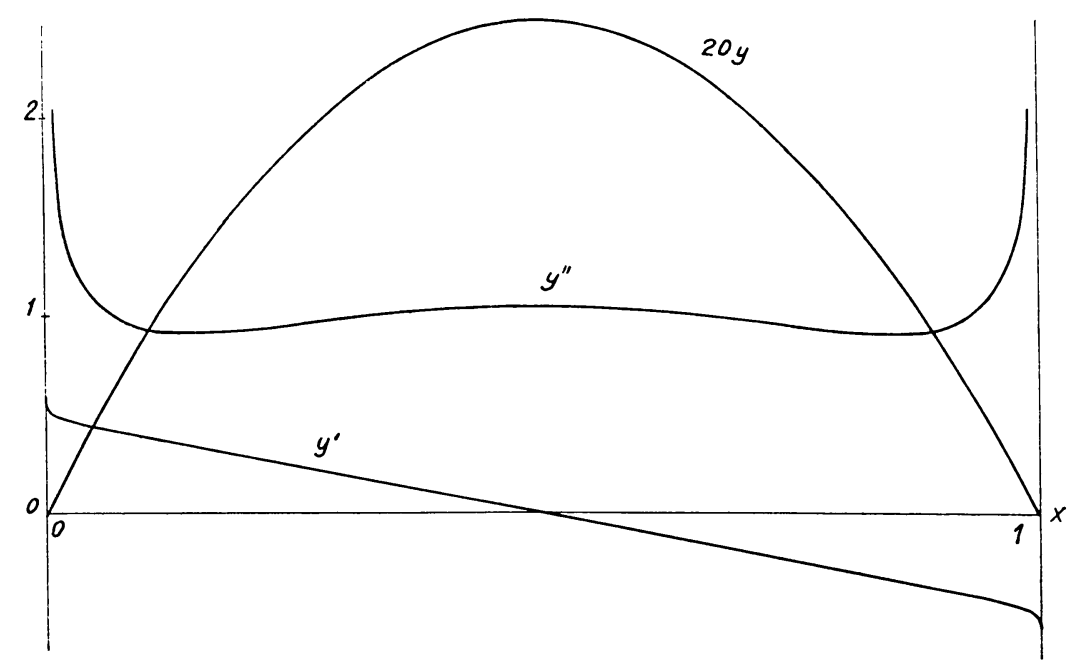

FIg. 1.

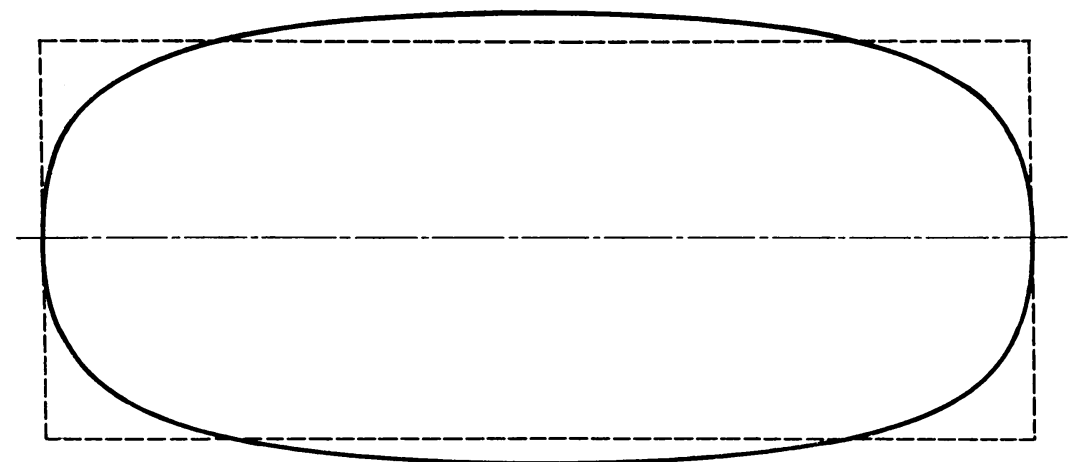

Fig. 2. 
6. Discussion. The variational method employed yields a stationary value of $\lambda$. Although there is little doubt that this value is a true maximum, a strict proof of this is lacking. Also, there is no proof concerning the supposed convergence of the sequence of functions obtained by the iteration formulas. We may hope that such proofs will eventually be given.

Following a very similar procedure one can solve the corresponding problem with other boundary conditions, such as clamped-free and clamped-clamped. In a paper to follow it will be shown that a whole group of related problems for beams and plates can be solved in a similar fashion.

\section{REFERENCES}

1. J. L. de Lagrange, Sur la figure des colonnes, Miscellanea Taurinensia 5 (1770-1773) 123

2. T. Clausen, Über die Form architektonischer Säulen, Bulletin physico-math. et astron. 1 (1849-1853) 279

3. J. B. Keller, The shape of the strongest column, Archive Ratl. Mech. Anal. 5 (1960) 275

4. I. Tadjbakhsh and J. B. Keller, Strongest columns and isoperimetric inequalities for eigenvalues, Journal Appl. Mech. 29 (1962) 159

5. P. R. Beesack, Isoperimetric inequalities for the nonhomogeneous clamped rod and plate, Journal Math. Mech. 8 (1959) 471

6. B. Schwarz, On the extrema of frequencies of nonhomogeneous strings with equimeasurable density, Journal Math. Mech. 10 (1961) 401

7. B. Schwarz, Some results on the frequencies of nonhomogeneous rods, Journal Math. Anal. and Appl. 5 (1962) 169

8. B. Schwarz, Bounds for the principal frequency of nonuniformly loaded strings, Israel Journal Math. 1 (1963) 11 